\title{
Insulator-quantum Hall liquid transitions in a two-dimensional electron gas using self-assembled InAs dots
}

\author{
Gil-Ho Kimª, , , J.T. Nicholls ${ }^{\mathrm{a}}$, C.-T. Liang ${ }^{\mathrm{a}, \mathrm{c}}$, D.A. Ritchie ${ }^{\mathrm{a}}$, S.I. Khondaker ${ }^{\mathrm{a}}$

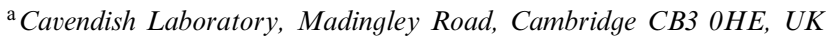 \\ ${ }^{\mathrm{b}}$ Telecommunication Basic Research Laboratory, ETRI, Yusong, P.O. Box 106, Daejon 305-600, South Korea \\ ${ }^{\mathrm{c}}$ Department of Physics, National Taiwan University, Taipei 106, Taiwan
}

\begin{abstract}
We investigate the transport properties of two-dimensional electron gases (2DEG) formed in a GaAs/AlGaAs quantum well, where self-assembled InAs quantum dots were grown at the center of the GaAs well. Due to the resulting strain fields repulsive short-range scattering is experienced by the conduction electrons in the 2DEG. In a perpendicular magnetic field, there are transitions between quantum Hall liquids at filling factors $v=1$ and 2 and the insulating phase. We show that the boundary of insulator-Quantum Hall transitions can be identified either by analysing the temperature-independent points in $\rho_{x x}$ or from the peaks in $\sigma_{x x}$ at low temperatures and both methods give similar results. (C) 2002 Elsevier Science B.V. All rights reserved.
\end{abstract}

PACS: 73.40.Hm; 72.10.Fk; 74.25.Fy

Keywords: Self-assembled quantum dots; Quantum Hall effect; Spin splitting

In a perpendicular magnetic field $B$, a twodimensional electron gas (2DEG) exhibits the quantum Hall $(\mathrm{QH})$ effect. There are localised states in the Landau level (LL) tails and extended states at the LL centres, and when the magnetic field $B$ is decreased at a fixed carrier density $(n)$, the localised and extended states alternately move down through the Fermi energy. It is believed [1,2] that at zero field a $2 \mathrm{DEG}$ becomes insulating, and therefore the extended states at the centre of each LL "float" up in energy as $B \rightarrow 0$.

Measurements [3-5] of disordered 2DEGs in GaAs show a transition with increasing $B$ from a strongly

\footnotetext{
* Corresponding author. Telecommunication Basic Research Laboratory, ETRI, Yusong, P.O. Box 106, Daejon 305-600, South Korea. Tel.: +82-42-860-1149; fax: +82-42-860-6836.

E-mail address: ghk@etri.re.kr (G.-H. Kim).
}

localised zero-field insulating phase into a $\mathrm{QH}$ liquid of filling factor $v=2$, and at higher $B$ there is a transition back to the insulating phase. The observation of these insulator- $\mathrm{QH}$ liquid transitions enables one to construct the phase diagram in $n-B$ space which looks like the theoretically expected global phase diagram proposed by Kivelson et al. [6]. However in later measurements $[8,9]$, transitions were observed with increasing $B$ from an insulator $\rightarrow v=2 \mathrm{QH}$ liquid $\rightarrow v=1 \mathrm{QH}$ liquid $\rightarrow$ insulator $(0-2-1-0)$ as $B$ was increased, and not the expected $(0-1-2-1-0)$ transitions. Pioneering experimental results on a Si electron gas show $(0-6-0)$ and $(0-2-0-1-0)$ transitions which are also not consistent with the global phase diagram [7].

In this paper, we present a new 2D system for observing insulator-QH transitions. We show that the 
boundary of insulator- $\mathrm{QH}$ transitions can be identified either by analysing the temperature-independent points in $\rho_{x x}$ or from the peaks in $\sigma_{x x}$ at low temperatures and both methods give similar results.

The sample investigated was grown by molecular beam epitaxy. The structure consists of a $0.6 \mu \mathrm{m}$ thick undoped GaAs buffer layer, followed by a $500 \AA$ undoped $\mathrm{Al}_{0.33} \mathrm{Ga}_{0.67} \mathrm{As}$ barrier, a $200 \AA$ undoped GaAs quantum well, a $400 \AA$ undoped $\mathrm{Al}_{0.33} \mathrm{Ga}_{0.67} \mathrm{As}$ spacer layer, a $400 \AA \mathrm{Si}$-doped $\left(1 \times 10^{18} \mathrm{~cm}^{-3}\right)$ $\mathrm{Al}_{0.33} \mathrm{Ga}_{0.67}$ As layer, and finally a $170 \AA$ GaAs capping layer. During a growth interrupt an InAs layer with a coverage of 2.15 monolayers (ML) was grown (Stranski-Krastanov growth) into the central part of the GaAs quantum well. In sample C1335, 2.15 ML of InAs were followed by a $50 \AA$ GaAs capping layer; the InAs formed self-assembled quantum dots, having a density of $3.0 \times 10^{9} \mathrm{~cm}^{-2}$. We find the average dimension of the dots to be $\sim 280 \AA$ wide and $\sim 40 \AA$ high in this sample. All dots show strain contrast, and very few show loss of coherency, as observed [10]. For this sample, the ratio of the transport to quantum lifetime is approximately five, which is a consequence of short-range scattering from InAs dots [11].

Fig. 1(a) and (b) show longitudinal magnetoresistivity traces $\rho_{x x}(B)$ over the temperature range $T=$ $20-580 \mathrm{mK}$, for two different gate voltages $V_{\mathrm{g}}$. As $V_{\mathrm{g}}$ is made less negative, the effective disorder decreases and the zero-filed resistivity drops from being greater than $60-20 \mathrm{k} \Omega$. As in previous studies [5], the temperature independence of $\rho_{x x}(B)$ at a particular magnetic field and gate voltage $V_{\mathrm{g}}$, is used to identify the boundaries between different $\mathrm{QH}$ liquids at $v=1$ and 2, and the insulating phase (0). Fig. 1(a) shows $0-2-0$ transitions at $V_{\mathrm{g}}=-0.278 \mathrm{~V}$, which are identified by temperature independent $\rho_{x x}(B)$ at $B=1.2$ and $1.7 \mathrm{~T}$ (labelled $\mathrm{C}_{2}$ and $\mathrm{C}_{*}$ ), at which $\rho_{x x} \approx h / 2 e^{2}$. The $\rho_{x x}$ minimum at a magnetic field of $B \simeq 1.4 \mathrm{~T}$ correspond to the spin-degenerate filling factor $v=2$. At the higher carrier density, $V_{\mathrm{g}}=-0.260 \mathrm{~V}$ [Fig. 1(b)], proper zeros in the low temperature $\rho_{x x}(B)$ traces have developed at filling factors $v=1$ and 2, which are accompanied by $\mathrm{QH}$ plateaus in $\rho_{x x}(B)$. The $0-2$ transition $\left(\mathrm{C}_{2}\right)$ at $B=1.1 \mathrm{~T}$, and the $1-0$ transition $\left(\mathrm{C}_{1}\right)$ at $3.8 \mathrm{~T}$ are clearly defined because they separate phases of opposite temperature dependence.

In Fig. 2 we plot the magnetic field dependence of the conductivities $\sigma_{x x}$ (solid lines) and $\sigma_{x y}$ (dotted
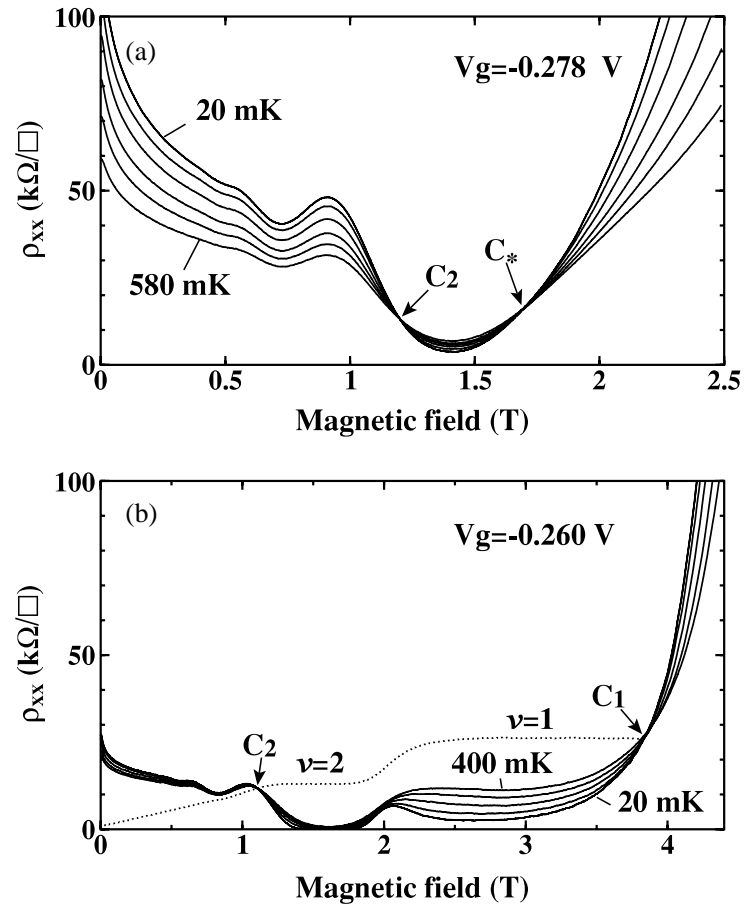

Fig. 1. $\rho_{x x}$ as a function of magnetic field at temperatures $20 \mathrm{mK} \leqslant T \leqslant 580 \mathrm{mK}$. The dotted line in (b) shows $\rho_{x y}$ at $T=297 \mathrm{mK}$.

lines) for a range of negative gate bias at $20 \mathrm{mK}$. At the most negative bias, $V_{\mathrm{g}}=-0.284 \mathrm{~V}$ [Fig. 2(a)] the peaks in $\sigma_{x x}$ have almost merged into a single feature. At $V_{\mathrm{g}}=-0.280 \mathrm{~V}$ [Fig. 2(b)] and $V_{\mathrm{g}}=-0.276 \mathrm{~V}$ [Fig. 2(c)], $\sigma_{x x}$ has split into two peaks, the minimum between corresponding to the $v=2$ spin-degenerate quantum Hall state. By $V_{\mathrm{g}}=-0.272 \mathrm{~V}$ [Fig. 2(d)] one begins to see the development in $\sigma_{x x}$ at higher magnetic fields of the spin-polarised $v=1$ state whilst $\sigma_{x y}$ has a well-quantised plateau $2 e^{2} / h$ at $v=2$. At a bias of $V_{\mathrm{g}}=-0.264 \mathrm{~V}$ [Fig. 2(e)] we observe the maxima (delocalised states) in $\sigma_{x x}$ and strong quantised plateaus at $2 e^{2} / h$ and $e^{2} / h$ in $\sigma_{x y}$.

Transition points on the phase boundaries in Fig. 3(a) were obtained from the temperatureindependent points in the $\rho_{x x}$ data. As may be seen, all of the states are insulating at zero field. The second phase diagram utilises the fact that the peaks in $\sigma_{x x}$ are a signature for the delocalised states [12]. Fig. 3(b) shows the phase diagram which derived from the conductance peaks in $\sigma_{x x}$ at $20 \mathrm{mK}$. The 


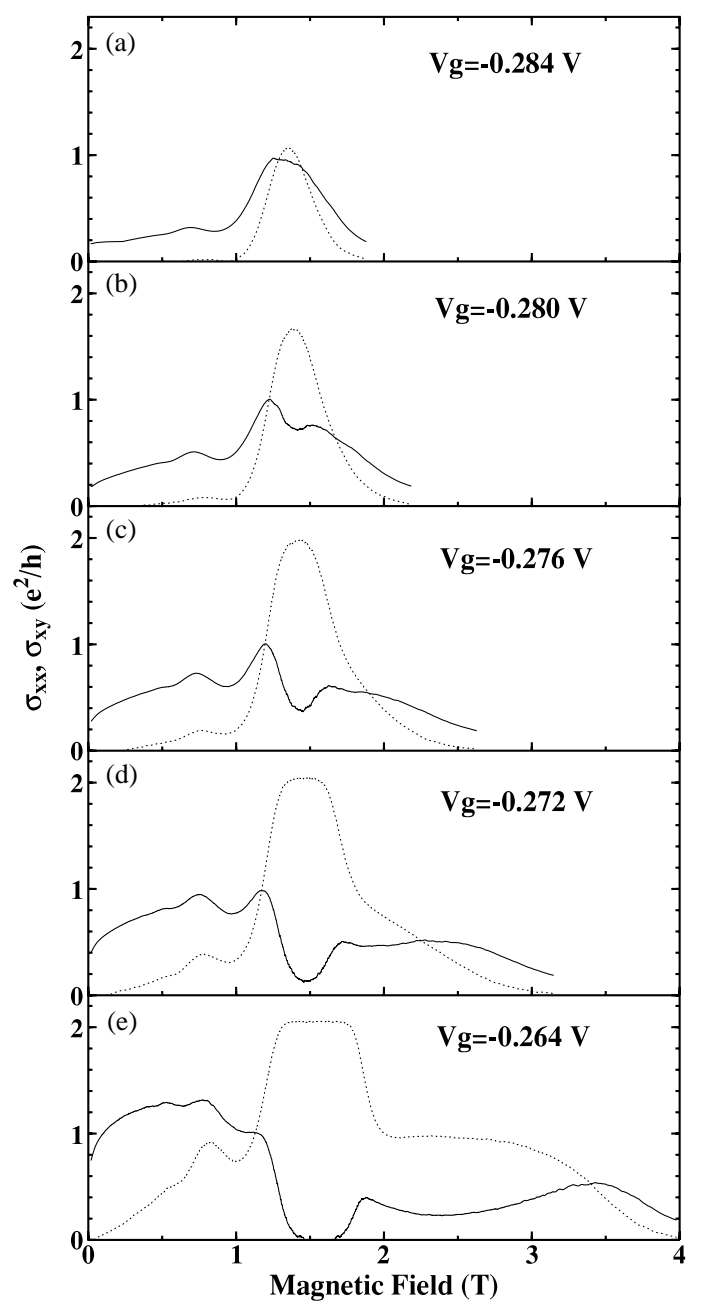

Fig. 2. $\sigma_{x x}$ (solid lines) and $\sigma_{x y}$ (dotted lines) as function of magnetic field for various gate voltages at $20 \mathrm{mK}$. The conductivities $\sigma_{x x}$ and $\sigma_{x y}$ are calculated from the resistivity $\rho_{x x}$ and $\rho_{x y}$ via the matrix relation.

exception being the closed triangle data points which were obtained at a temperature of $1.2 \mathrm{~K}$.

Fogler and Shklovskii [13] have recently proposed that the collapse of spin splitting in the quantum Hall effect is due to the disorder-induced broadening of Landau levels. Fig. 3(b) shows the disorder-induced collapse of spin splitting. The similarity of Fig. 3(a) and (b) show that the phase boundary can be identified either by analysing the temperature-independent points in $\rho_{x x}$ or from the peaks in $\sigma_{x x}$ at low temperatures $(<300 \mathrm{mK})$.
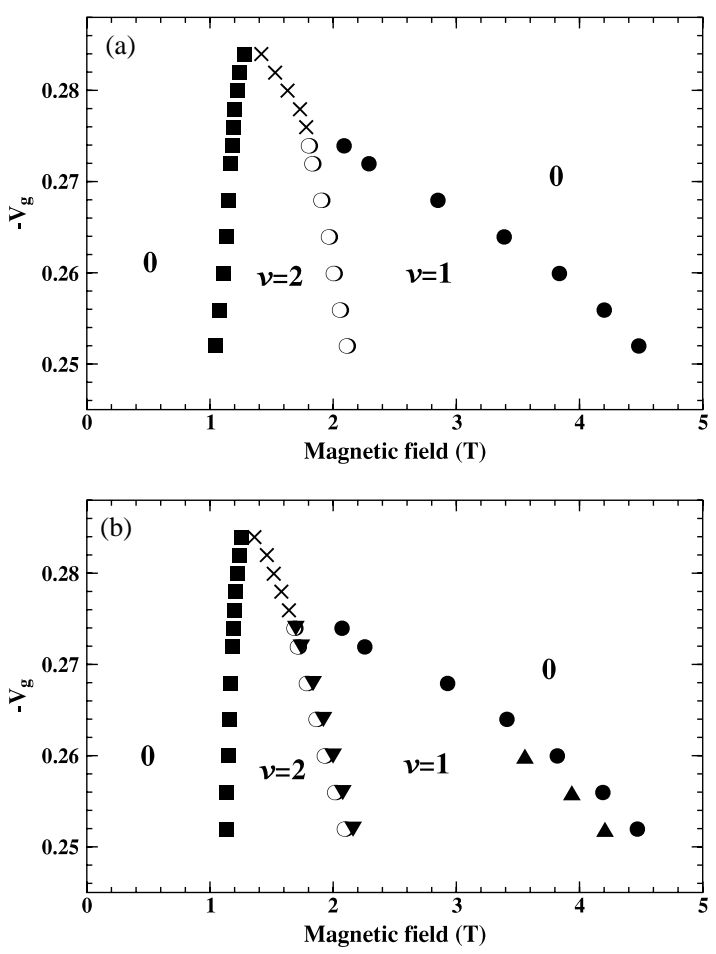

Fig. 3. The phase diagram determined from (a) temperatureindependent points in $\rho_{x x}$ traces and (b) peaks in $\sigma_{x x}$. Symbols indicate transitions from (i) $0-2$ (solid squares), (ii) 2-0 (crosses), (iii) $2-1$ (open circles), (iv) 1-0 (solid circles), and (v) peaks in $\sigma_{x x}$ at $1.2 \mathrm{~K}$ (solid triangles).

In summary, transport measurements of InAs self-assembled quantum dot samples show insulatorQH transitions. The transport result of the quantum Hall transitions can be explained by assuming that the self-assembled InAs structures introduce strong scattering in the two-dimensional electron gas. For the first time, we have shown that phase diagrams in the $\mathrm{QH}$ effect can be constructed either by analysing the temperature-independent points in $\rho_{x x}$ or from the peaks in $\sigma_{x x}$ at low temperatures $(<300 \mathrm{mK})$. We note that both methods indeed give similar phase diagrams.

The authors wish to thank the EPSRC UK for supporting this work. Gil-Ho Kim acknowledges support from the Arkinson Fund, Clare College, Cambridge and the Korean Ministry of Information and Communication. C.-T. Liang is grateful for financial support from the NSC, Taiwan. 


\section{References}

[1] D. Khmelnitskii, Phys. Lett. A 106 (1984) 182.

[2] R.B. Laughlin, Phys. Rev. Lett. 52 (1984) 2304.

[3] H.W. Jiang, C.E. Johnson, K.L. Wang, S.T. Hannahs, Phys. Rev. Lett. 71 (1993) 1439.

[4] T. Wang, K.P. Clark, G.F. Spencer, A.M. Mack, W.P. Kirk, Phys. Rev. Lett. 72 (1994) 709.

[5] R.J.F. Hughes, J.T. Nicholls, J.E.F. Frost, E.H. Linfield, M. Pepper, C.J.B. Ford, D.A. Ritchie, G.A.C. Jones, E. Kogan, M. Kaveh, J. Phys.: Condens. Matter 6 (1994) 4763.

[6] S.A. Kivelson, D.H. Lee, S.C. Zhang, Phys. Rev. B 46 (1992) 2223.
[7] V.M. Pudalov, M. D’Iorio, J.W. Campbell, JETP Lett. 57 (1993) 608.

[8] D. Shahar, D.C. Tsui, J.E. Cunningham, Phys. Rev. B 52 (1995) 14372.

[9] G.H. Kim, J.T. Nicholls, S.I. Khondaker, I. Farrer, D.A. Ritchie, Phys. Rev. B 61 (2000) 10910.

[10] G.D. Lian, J. Yuan, L.M. Brown, G.H. Kim, D.A. Ritchie, Appl. Phys. Lett. 73 (1998) 49.

[11] G.H. Kim, D.A. Ritchie, M. Pepper, G.D. Lian, J. Yuan, L.M. Brown, Appl. Phys. Lett. 73 (1998) 2468.

[12] I. Glozman, C.E. Johnson, H.W. Jiang, Phys. Rev. Lett. 74 (1995) 594.

[13] M.M. Fogler, B.I. Shklovskii, Phys. Rev. B 52 (1995) 17366. 